\title{
Challenges Track Chairs' Welcome
}

It is our great pleasure to welcome you to the WWW 2018 Challenges Track. It is the first time that the WWW conference includes such a track, which aim was to showcase the maturity of the state of the art on tasks common to the Web community and adjacent academic communities, in a controlled setting of rigorous evaluation. Through our call for challenge organisation, we also wanted to see which open questions might be seen as most relevant in this community today, and how groups of researchers might come together around shared resources (e.g. datasets) to address those questions in a hands-on, practical way. We received five proposals for challenges, with very varied topics. We selected four of them, which were proposed by well established researchers in their respective domains:

- Learning to Recognise Musical Genre from Audio, organised by Michaël Defferrard, Sharada Mohanty, Sean Carroll and Marcel Salathé

- Knowledge Extraction for the Web of Things (KE4WoT), organised by Amelie Gyrard, Mihaela Juganaru-Mathieu, Manas Gaur, Swati Padhee and Amit Sheth

- Question Answering Mediated by Visual Clues and Knowledge Graphs, organised by Fabrício Faria, Andre Freitas, Tingting Mu, Alessio Sarullo and Ricardo Usbeck

- Multi-lingual Opinion Mining and Question Answering over Financial Data, organised by Andre Freitas, Manel Zarrouk and Brian Davis

As can be seen, the topics addressed varied from being purely focused on a domain specific task, without constraints on the technical approach to take (e.g. music genre recognition) to fundamentally technical tasks, which can be seen as useful across domains (question answering), with two of the challenges representing a mix of both.

While the four challenges varied a lot in the way they attracted participation, all challenge organisers put in a great effort in setting up the tasks for their challenge, creating in the process shared resources (datasets, evaluation methods, etc.) of value in their respective area, and in gathering a community around those tasks. The concrete practical value of the solutions proposed by participants to those contests represents a very interesting way to progress on such complex challenges, as do all the different elements involved in the organisation of the challenges as part of the conference (from the formulation of the specific problem addressed, to the connection made across the community through different contributions).

During the conference, each of the challenges will be presented as part of two dedicated sessions on Friday April 27th 2018. The organisers of each challenge will have 40 Minutes to present an overview of their challenge, of the associated tasks and of the created resources. They will also include, when relevant, presentations from the top participants in their challenge, and announce the challenge winners.

Mathieu d'Aquin

Challenges Track Chair

Data Science Institute,

National University of

Ireland Galway

Insight Centre for Data

Analytics, Ireland

\section{Elena Cabrio \\ Challenges Track Chair \\ Wimmics research team \\ Université Côte d'Azur, Inria, \\ CNRS, I3S, France}

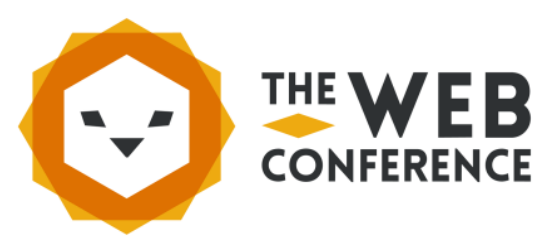

